\title{
Mutational heterogeneity of angioimmunoblastic T-cell lymphoma indicates distinct lymphomagenic pathways
}

\author{
Mathijs Willemsen (1)', Myrurgia Abdul Hamid', Bjorn Winkens² and Axel zur Hausen ${ }^{1}$
}

The 2016 revision of the World Health Organization classification of lymphoid neoplasms introduces the umbrella category "nodal T-cell lymphomas with Tfollicular helper $\left(\mathrm{T}_{\mathrm{FH}}\right)$ phenotype", which includes angioimmunoblastic T-cell lymphoma (AITL), follicular T-cell lymphoma and nodal peripheral T-cell lymphoma (PTCL) with a $\mathrm{T}_{\mathrm{FH}}$ phenotype ${ }^{1}$. One of the genetic features clustering $\mathrm{T}_{\mathrm{FH}}$ cell-derived lymphomas is a recurrent $R H O A$ G17V mutation, which is present in approximately $60 \%$ of investigated cases $^{2,3}$. RHOA is a member of the Rho family of GTPases which function as molecular regulators of diverse cellular functions ${ }^{4}$. Mutant RHOA acts as a dominant-negative signaling protein sequestering guanine nucleotide exchange factors (GEFs) thereby inhibiting wildtype RHOA and potentially other GEF-dependent proteins ${ }^{3}$. In vivo, mutant $R H O A$ has recently been shown to skew $\mathrm{CD}_{4}^{+} \mathrm{T}$-cell differentiation towards the $\mathrm{T}_{\mathrm{FH}}$ lineage and promote AITL lymphomagenesis ${ }^{5}$. Thus, the RHOA G17V mutation can be viewed as pivotal genetic aberration in AITL and potentially other $\mathrm{T}_{\mathrm{FH}}$ cell-derived lymphomas. Mutations contributing to lymphomagenesis in wildtype $R H O A$ AITL cases remain largely unknown. This mutational heterogeneity points towards the existence of distinct AITL lymphomagenic pathways. In this report, we explore the mutational landscape of AITL by assessing the data from large sequencing studies focusing on the association between RHOA mutational status and recurrent

\footnotetext{
Correspondence: Mathijs Willemsen (mathijs.willemsen@mumc.nl)

'Department of Pathology, GROW-School for Oncology \& Developmental Biology, Maastricht University Medical Centre, Maastricht, The Netherlands

${ }^{2}$ Department of Methodology and Statistics, CAPHRI-Care and Public Health Research Institute, Maastricht University, Maastricht, The Netherlands
}

mutations in other genes to provide evidence for the existence of distinct lymphomagenic pathways in AITL.

Sequencing studies of AITL and/or PTCL published between 01-01-2014 and 28-02-2017 using an English language restriction were identified with PubMed. In total, 117 abstracts were screened. Only 34 articles were eligible for full text review. Studies were included in our analysis if they contained ten or more AITL cases and used targeted deep sequencing of RHOA, TET2, $D N M T 3 A, I D H 2, C D 28$ and multiple other genes or whole genome/exome/transcriptome approaches. Also, the original dataset had to be available to the authors. Five of the 34 articles met the prespecified inclusion criteria and were included in our analysis ${ }^{6-10}$. The article selection process was performed by two authors.

In total, these studies analyzed 239 AITL cases using various sequencing techniques. Of interest, in 13.8\% (33/ 239) of investigated AITL cases no detectable mutations were reported. RHOA was mutated in $61.1 \%(146 / 239)$ of the investigated AITL cases. The remaining 25.1\% (60/ 239) of cases were wildtype, but carried mutations in other genes (Table 1a).

We focused on the data extract of all wildtype $R H O A$ AITL cases to identify potentially recurrent mutations contributing to AITL lymphomagenesis other than RHOA. Only mutations occurring in more than $5 \%$ of targeted cases and identified in two or more studies were classified as recurrent. TET2, CD28, DNTM3A, PLCG1, IDH2, VAV1, FYN and STAT3 were mutated in 60.7\% (34/56), 18.6\% (8/43), 17.9\% (10/56), 14.0\% (6/43), 13.8\% (8/58), $11.6 \%$ (5/43), 7.8\% (4/51) and 7.0\% (3/43) of targeted wildtype RHOA AITL cases, respectively (Supplementary Data set 1 ). As these mutations also frequently 
Table 1 RHOA mutational status of included studies (A) and association with recurrent mutations in wildtype RHOA AITL cases (B)

\begin{tabular}{|c|c|c|c|c|c|}
\hline (A) Author & Sequencing method & AITL cases & $\begin{array}{l}\text { No detectable } \\
\text { mutations }\end{array}$ & Wildtype $R H O A$ & Mutant $R H O A^{\mathrm{a}}$ \\
\hline Abate et al. ${ }^{6}$ & RNA-seq, TDS (VAV1) & 60 & 15 & 20 & 25 \\
\hline Nguyen et al. ${ }^{7} 2017$ & TDS (71 genes) & 48 & 6 & 9 & 33 \\
\hline Vallois et al. ${ }^{9}$ & TDS (69 genes) & 72 & 8 & 18 & 46 \\
\hline Yoo et al. ${ }^{10 \mathrm{~b}}$ & TDS (70 genes) & 29 & 3 & 5 & 21 \\
\hline Palomero et al. ${ }^{8 c}$ & $\begin{array}{l}\text { RNA-seq, TDS (13 genes), allele- } \\
\text { specific PCR (RHOA) }\end{array}$ & 30 & 1 & 8 & 21 \\
\hline Total & & 239 & $33(13.8 \%)$ & $60(25.1 \%)$ & $146(61.1 \%)$ \\
\hline (B) Gene & Mutant $R H O A$ AITL cases/total ${ }^{a}$ & $\begin{array}{l}\text { Wildtype } R H O A \text { AITL } \\
\text { cases/total }^{d}\end{array}$ & Odds ratio & $95 \% \mathrm{Cl}$ & $p$-value \\
\hline TET2 $2^{c, ~ e ~}$ & $101 / 146(69.2 \%)$ & $33 / 93(35.5 \%)$ & 3.46 & $1.92,6.22$ & $<0.001$ \\
\hline DNMT3A $A^{c, e}$ & $34 / 146(23.3 \%)$ & 10/93 (10.8\%) & 2.14 & $0.99,4.66$ & 0.076 \\
\hline$I D H 2^{c, ~ e ~}$ & $51 / 146$ (34.9\%) & 9/93 (9.7\%) & 6.68 & $2.89,15.45$ & $<0.001$ \\
\hline $\mathrm{CD} 28$ & $14 / 92(15.2 \%)$ & $7 / 69(10.1 \%)$ & 1.73 & $0.64,4.73$ & 0.399 \\
\hline $\operatorname{CD} 28^{\mathrm{f}}$ & $24 / 92(26.1 \%)$ & $8 / 69(11.6 \%)$ & 2.60 & $0.98,6.86$ & 0.093 \\
\hline FYN & $5 / 113(4.4 \%)$ & $3 / 78(3.8 \%)$ & 1.38 & $0.30,6.29$ & 0.972 \\
\hline PLCG1 & $6 / 92(6.5 \%)$ & $6 / 69(8.7 \%)$ & 0.85 & $0.26,2.85$ & 0.960 \\
\hline STAT3 & $2 / 92(2.2 \%)$ & $3 / 69(4.3 \%)$ & 0.68 & $0.13,3.69$ & 0.979 \\
\hline VAV1 & $2 / 92(2.2 \%)$ & $5 / 69(7.2 \%)$ & 0.27 & $0.046,1.56$ & 0.268 \\
\hline
\end{tabular}

TDS targeted deep sequencing, $\mathrm{Cl}$ confidence interval

ancluding non-G17V RHOA mutations

${ }^{b}$ Only 29/45 AITL cases were analyzed using TDS

'Only 30/35 AITL cases were analyzed by allele-specific PCR and targeted deep sequencing. Only one case showed no mutations in both techniques (no detectable mutations). RNA sequencing data not included due to uncertainty of diagnosis

${ }^{\mathrm{d}}$ Including cases with no detectable mutations

e Missing data regarding mutational status of TET2 $(n=14), D N M T 3 A(n=14)$ and IDH2 $(n=10)$ from Vallois et al. included as not mutated

fIncluding cases with CTLA4-CD28 gene fusion

occur in mutant RHOA AITL cases, we performed Mantel-Haenszel statistics to assess the association between these mutations and RHOA mutational status across different studies (SPSS v21 IBM Corp., Armonk, NY, USA). A $p$-value $\leq 0.05$ was considered significant.

Statistical analysis showed that mutations in TET2 and $I D H 2$ were associated with mutant $R H O A$ status $(p<$ 0.001 for both genes). Mutations in DNMT3A and CD28, including CTLA4-CD28 fusion, also tend to show this association ( $p=0.076$ and 0.093). Interestingly, despite being mutated in a low number of cases, mutations in $V A V 1$ tend to associate with wildtype RHOA status ( $p=$ 0.268). Mutations in FYN, PLCG1 and STAT3 showed no significant association with $R H O A$ mutational status $(p=$ 0.972, 0.960 and 0.979) (Table 1b).

This study reports on the association between $R H O A$ mutational status and other recurrent mutations in AITL. We found an association between mutant RHOA and mutations in TET2 and IDH2. Despite being mutually exclusive in acute myeloid leukemia, mutations in $I D H 2$ and TET2 tend to co-occur in $A I T L^{11}$. Gene expression profiling and promoter methylation analysis of double mutant AITL cases showed upregulation of genes associated with $\mathrm{T}_{\mathrm{FH}}$ phenotype and downregulation of genes associated with $\mathrm{T}_{\mathrm{H}} 1$ phenotype ${ }^{11}$. Mutant $I D H 2$ and TET2 potentially cooperate with mutant $R H O A$ to induce a potent $\mathrm{T}_{\mathrm{FH}}$ phenotype in vivo. This mechanism would explain the association found between these mutations in the present study. We also identified a strong tendency towards association between mutant RHOA and mutations in DNMT3A. The exact mechanism by which mutations in epigenetic modifiers contribute to lymphomagenesis remain to be elucidated, but alterations in hematopoietic stem cell differentiation is an attractive theory. The present study also identified a strong tendency towards association between mutant $R H O A$ and mutations in $C D 28$, including CTLA4-CD28 gene fusion. CD28 mutations in AITL are confined to hotspot residues 


\section{Angioimmunoblastic T-cell lymphoma}

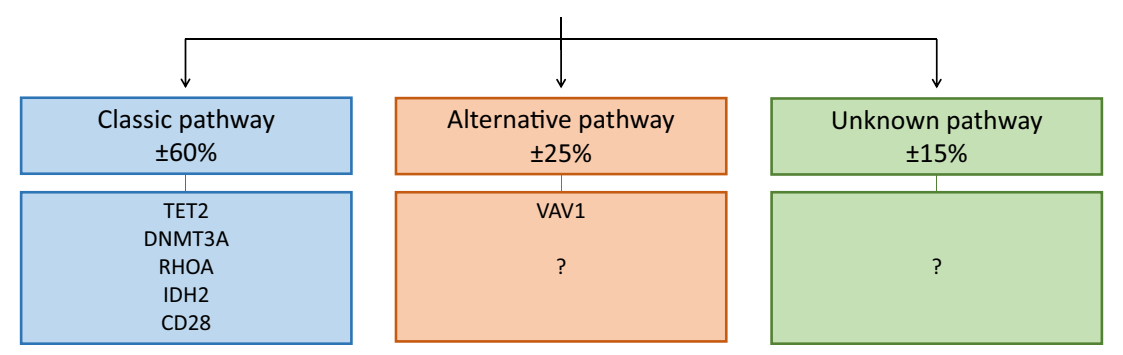

Fig. 1 Three distinct lymphomagenic pathways in AITL. Our statistical analysis has identified three potentially distinct lymphomagenic pathways in AITL. The classic pathway accounts for approximately $60 \%$ of AITL cases and is characterized by the RHOA G17V mutation in association with several other recurrent mutations. The alternative pathway accounts for approximately $25 \%$ of AITL cases and is characterized by mutations in VAV1 or potentially other members of the Rho family of GTPases or their regulatory proteins. The mutations that drive the remaining approximately $15 \%$ of AITL cases are unknown. It is possible that mutations in signaling pathways directly regulating $T_{F H}$ differentiation contribute to these cases

D124 and T195 and render CD28 constitutively active $^{10,12}$. The CTLA4-CD28 fusion gene has only been reported in an Asian cohort $^{10}$. Therefore, validation of this fusion gene in other cohorts is essential to confirm the association between mutant $R H O A$ and mutations in $C D 28$. Altogether, these findings point towards a classic AITL lymphomagenic pathway (Fig. 1). Several therapeutic approaches targeting epigenetic modifiers, IDH2 or $\mathrm{CD} 28$ are currently in clinical trials or have already been approved for other diseases ${ }^{13,14}$. The tendency of these mutations to cluster will potentially help to develop novel combinatorial therapeutic regimens.

Despite being mutated in rather a low number of AITL cases, this study identified the tendency of mutations in $V A V 1$ to associate with wildtype RHOA. VAV1 encodes a Rho GTPase family-specific GEF which is primarily expressed in the hematopoietic system ${ }^{15}$. The studies that targeted VAV1 identified three missense mutations (E524D, E556D and D797G), two frameshift deletions (151_158del and 778_783del), one fusion gene (VAV1S100A7) and one in-frame deletion $\left(778 \_786 \mathrm{del}\right)^{6,9,10}$. Abate et al. found the 778 -786 in-frame deletion and VAV1-S100A7 fusion gene to be locked in a constitutively active conformation, indicated by high levels of Tyr174 phosphorylation ${ }^{6}$. Both genetic aberrations resulted in increased VAV1 catalytic-dependent functions downstream of RAC1, another member of the Rho family of GTPases ${ }^{6}$. These findings are in accordance with previous experiments showing that constitutively active VAV1 predominantly increases nucleotide exchange of RAC1 and to a lesser extend of $\mathrm{RHOA}^{16}$. Interestingly, the RAC1 pathway is upregulated in mutant $R H O A$ compared to wildtype RHOA AITL cases, providing evidence that both mutations have similar effects on VAV1 catalyticdependent pathways ${ }^{17}$. Additionally, Abate et al. found that the VAV1-S100A7 fusion gene resulted in increased NFAT activity, a functional readout of VAV1 non- catalytic activity, whereas both the $778 \_786$ in-frame deletion and VAV1-S100A7 fusion gene increased expression of NFAT target genes ${ }^{6}$. A recently published study, not yet indexed by PubMed at the time of our search, identified activating VAV1 mutations in $8.2 \%$ (7/ $85)$ of wildtype $R H O A$ AITL cases, compared to $0 \%(0 / 41)$ in mutant $R H O A$ AITL cases, respectively ${ }^{18}$. They also showed that mutant RHOA enhances the non-catalytic functions of VAV1 through increased Tyr174 phosphorylation, thereby increasing NFAT activity and expression of NFAT target genes. Together, these data not only strengthen the association between mutant $V A V 1$ and wildtype $R H O A$, but also provide evidence that mutant RHOA and mutant VAVI have similar effects on catalytic and non-catalytic signaling pathways downstream of VAV1. Therefore, we deduce from these data that mutant RHOA and mutant VAV1 contribute to AITL lymphomagenesis in a similar manner. This would mean that $V A V 1$ is part of an alternative AITL lymphomagenic pathway (Fig. 1). Previous clinicopathological studies have shown that mutant RHOA AITL cases have worse performance status, more frequent $\mathrm{B}$-symptoms and splenomegaly and a more potent $\mathrm{T}_{\mathrm{FH}}$ immunophenotype compared to wildtype $R H O A$ AITL cases ${ }^{19,20}$. These data provide additional justification for separating AITL subgroups.

According to our analysis, no mutations were detectable in approximately $15 \%$ (range 3-25\%) of AITL cases (Fig. 1). Exploring the mutational landscape of AITL using targeted deep sequencing panels enriched with members of the Rho family of GTPases and their regulatory proteins might identify driver mutations in this subgroup. It is also possible that other lymphomagenic mechanisms contribute to some AITL cases, for example mutations in signaling pathways directly regulating $\mathrm{T}_{\mathrm{FH}}$ differentiation.

We are aware that there are some limitations to our study. Our findings are entirely based on retrospective 
data from a relatively small sample size. Furthermore, there is significant technical heterogeneity between the sequencing studies from which the data is derived. The individual studies use different sequencing techniques, bioinformatics pipelines for data processing and mutation calling methods. Despite these limitations, this study remains noteworthy as it provides a unique perspective on associations and possible collaborations between the most common genetic aberrations in AITL as well as providing a rationale for future research.

In short, using data from large sequencing studies this study reports on varying associations between $R H O A$ mutational status and other recurrent mutations in AITL. These findings enable us to identify three potentially distinct AITL lymphomagenic pathways. First, the classic pathway with the RHOA G17V mutation which is associated with mutations in TET2, DNMT3A, IDH2 and $C D 28$. Secondly, the alternative pathway with mutations in $V A V 1$ or potentially yet unidentified mutations in members of the Rho family of GTPases or their regulatory proteins. Third, AITL cases with unknown mutations which might arise from direct mutations in pathways regulating $\mathrm{T}_{\mathrm{FH}}$ differentiation. To what extend these different lymhpomagenic pathways result in different clinical behavior of AITL is largely unknown. Additional evidence on the mutational landscape of AITL, especially wildtype RHOA AITL cases, is needed to either confirm or refute our findings. Furthermore, prospective data is needed to identify potential clinical differences between the distinct lymphomagenic pathways of AITL proposed in this manuscript.

\section{Authors' contributions}

M.W. developed the initial hypothesis that lead to this work and wrote the manuscript. M.A.H. and A.Z.H. were involved in critical reviewing and revising the manuscript. B.W. supervised the statistical analysis.

\section{Conflict of interest}

The authors declare that they have no conflict of interest.

\section{Publisher's note}

Springer Nature remains neutral with regard to jurisdictional claims in published maps and institutional affiliations.

Supplementary information accompanies this paper at (https://doi.org/ 10.1038/s41408-017-0047-2).
Received: 19 November 2017 Revised: 19 November 2017 Accepted: 29 November 2017

Published online: 17 January 2018

\section{References}

1. Swerdlow, S. H. et al. The 2016 revision of the World Health Organization classification of lymphoid neoplasms. Blood 127, 2375-2390 (2016).

2. Dobay, M. P. et al. Integrative clinicopathological and molecular analyses of angioimmunoblastic T-cell lymphoma and other nodal lymphomas of follicular helper T-cell origin. Haematologica 102, e148-e151 (2017).

3. Cools, J. RHOA mutations in peripheral T cell lymphoma. Nat. Genet. 46, 320-321 (2014)

4. Hodge, R. G. \& Ridley, A. J. Regulating Rho GTPases and their regulators. Nat. Rev. Mol. Cell. Biol. 17, 496-510 (2016).

5. Cortes, J. R. et al. Role and Mechanisms of Rhoa G17V in the Pathogenesis of AITL. Blood 128, 621 (2016). (Abstract 608).

6. Abate, F. et al. Activating mutations and translocations in the guanine exchange factor VAV1 in peripheral T-cell lymphomas. Proc. Natl. Acad. Sci. USA 114, 764-769 (2017)

7. Nguyen, T. et al. Identification of cell-type-specific mutations in nodal T-cell lymphomas. Blood Cancer J. 7, e516 (2017).

8. Palomero, T. et al. Recurrent mutations in epigenetic regulators, RHOA and FYN kinase in peripheral T cell lymphomas. Nat. Genet. 46, 166-170 (2014).

9. Vallois, D. et al. Activating mutations in genes related to TCR signaling in angioimmunoblastic and other follicular helper T-cell-derived lymphomas. Blood 128, 1490-1502 (2016).

10. Yoo, H. Y. et al. Frequent CTLA4-CD28 gene fusion in diverse types of $T$ cell lymphoma. Haematologica 101, 757-763 (2016).

11. Wang, C. et al. IDH2R172 mutations define a unique subgroup of patients with angioimmunoblastic T-cell lymphoma. Blood 126, 1741-1752 (2015).

12. Rohr, J. et al. Recurrent activating mutations of CD28 in peripheral T-cell lymphomas. Leukemia 30, 1062-1070 (2016).

13. Willemsen, M. \& Schouten, H. C. Inappropriate costimulation and aberrant DNA methylation as therapeutic targets in angioimmunoblastic T-cell lymphoma. Biomark. Res. 5, 6 (2017).

14. Schmitz, N. \& Leval, L. How I manage peripheral T-cell lymphoma, not otherwise specified and angioimmunoblastic T-cell lymphoma: current practice and a glimpse into the future. Br. J. Haematol. 176, 851-866 (2017).

15. Bustelo, X. R. Vav family exchange factors: an integrated regulatory and functional view. Small GTPases 5, e973757 (2014).

16. Crespo, P., Schuebel, K. E., Ostrom, A. A., Gutkind, J. S. \& Bustelo, X. R. Phosphotyrosine-dependent activation of Rac-1 GDP/GTP exchange by the vav proto-oncogene product. Nature 385, 169-172 (1997).

17. Manso, R. et al. The RHOA G17V gene mutation occurs frequently in peripheral T-cell lymphoma and is associated with a characteristic molecular signature. Blood 123, 2893-2894 (2014).

18. Fujisawa M. et al. Activation of RHOA-VAV1 signaling in angioimmunoblastic T-cell lymphoma. Leukemia. https://doi.org/10.1038/leu.2017.273 (2017).

19. Nagao, R. et al. Clinicopathologic analysis of angioimmunoblastic T-cell lymphoma with or without RHOA G17V mutation using formalin-fixed paraffinembedded sections. Am. J. Surg. Pathol. 40, 1041-1050 (2016).

20. Ondrejka, S. L. et al. Angioimmunoblastic T-cell lymphomas with the RHOA p. Gly17Val mutation have classic clinical and pathologic features. Am. J. Surg. Pathol. 40, 335-341 (2016). 